Pacific Journal of Mathematics

FULL ANALYTIC SUBSPACES FOR CONTRACTIONS WITH 


\title{
FULL ANALYTIC SUBSPACES FOR CONTRACTIONS WITH RICH SPECTRUM
}

\author{
SCOTT W. BROWN
}

\begin{abstract}
It is shown that contractions with sufficiently rich spectrum have special kinds of invariant subspaces. These subspaces are analogous to the Hardy space $\mathrm{H}^{2}$ when viewed as an invariant subspace for the contraction of multiplication by $z$ on $L^{2}$ (of the circle).
\end{abstract}

1. Introduction. This paper deals with the invariant subspace structure of certain contractions on a separable Hilbert space $\mathscr{H}$. If $S$ is an operator on $\mathscr{H}$ of norm one, then the left essential spectrum of $S, \sigma_{\text {le }}(S)$, is called dominating in the unit disc if almost every point of the unit circle (with resepct to arc length measure) is a nontangential limit of a sequence from $\sigma_{\text {le }}(S)$. Throughout this paper, it will be assumed that $S$ is a completely nonunitary contraction (i.e., $\|S\| \leq 1$ ) with $\sigma_{\text {le }}(S)$ dominating in the unit disc. A closed invariant subspace, $\mathscr{S}$, for $S$ will be called full analytic, if $S$ and $\mathscr{S}$ together satisfy two properties. The first is that $\mathscr{S}$ is vector space isomorphic to a subspace of the vector space, $H(U)$, of all functions analytic on the open unit disc, $U$. The second is that if $y \in \mathscr{S}$ is associated with $f_{y}(z) \in H(U)$ under this isomorphism, then $S y$ is associated with $z f_{y}(z) \in H(U)$, so $f_{S y}(z)=z f_{y}(z)$. The main result of this paper, Theorem 2, gives that $S$ has a closed invariant subspace that is full analytic.

The phenomenon of full analytic invariant subspaces occurs frequently in analysis. The classical example is displayed by the bilateral shift and the Hardy space $\mathrm{H}^{2}$. Even more generally, every subnormal operator has a full analytic subspace as was shown recently by Olin and Thomson in [7] (the domain of analyticity has to be appropriately altered). One can find a simpler proof of this result for just normal operators in Chapter IX of [3]. The result of this present paper, although it does not subsume the subnormal or measure theoretic results concerning analyticity and vice versa, was clearly suggested by the work of Olin and Thomson.

The main tool to be used is that of matricial factorization as developed by Bercovici, Foias, and Pearcy in [1]. They used these results together with J. Langsam [2] to prove (among other things) that the 
operator $S$ as described above is reflexive. Since the work of [1] does not fit precisely to what will be used here, a full development of the factorization needed will be given.

Olin and Thomson used their analytic results in [7] to show that every subnormal operator is reflexive. In this article, the cycle will be completed, and the reflexivity result of [2] will be obtained for $S$ via the analyticity result of Theorem 2. The work of Julian Sheung [9] suggests that there may be a theorem which covers simultaneously the analytic and reflexivity results of [2], [7] and those presented here. However, any attempt to find it may not be worth the effort, since the analysis of [9] shows how difficult things can get.

The basic set-up is as follows. The reader is referred to [5] for a more detailed description, although the notation used here more closely follows that of [4]. Let $\mathscr{A}$ be an ultraweakly closed subalgebra of $\mathscr{B}(\mathscr{H})$. The predual of $\mathscr{A}$, a quotient space of the space of trace class operators, will be denoted by $\mathscr{C}$. If $A \in \mathscr{A}$ and $C \in \mathscr{C}$, then $A$ evaluated (as a linear functional) at $C$ is represented by $[A](C)$. If $x, y \in \mathscr{H}$, then $x \otimes y$ will represent that element in $\mathscr{C}$ defined by $[A](x \otimes y)=\langle A x, y\rangle$ for all $A \in \mathscr{A}$, where $\langle\cdot, \cdot\rangle$ is the inner product used on $\mathscr{H}$.

From here on it will be assumed that $\mathscr{A}$ satisfies two properties. All results will be stated in terms of such an algebra $\mathscr{A}$, its predual $\mathscr{C}$, and a particular operator $T \in \mathscr{A}$. The connection between $\mathscr{A}$ and $S$ will be given shortly.

The first property satisfied by $\mathscr{A}$ is that of being dual algebra isomorphic to $H^{\infty}$. This property is now outlined. Details can be found in [4] and [5]. Let $m$ be Lebesgue arc length measure on the unit circle. The weak* (or $\sigma\left(L^{\infty}(m), L^{1}(m)\right)$ ) closure in $L^{\infty}(m)$ of the polynomials will be denoted $H^{\infty}$. Now $H^{\infty}$ has a weak* topology endowed on it from this structure. It will be assumed that there is a map $\Gamma:\left(H^{\infty}\right.$, weak $\left.{ }^{*}\right) \rightarrow$ $\left(\mathscr{A}\right.$, weak $\left.{ }^{*}\right)$ that is a homeomorphism, onto, an isometry, and an algebra isomorphism (i.e., $\Gamma$ is a dual algebra isomorphism). Throughout this paper $T$ will denote $\Gamma(z)$ where $z$ is the identity function on the unit circle.

The second condition to be placed on $\mathscr{A}$ is somewhat technical. It will be assumed that given any $C \in \mathscr{C}$ of norm $k$, there exist sequences $\left\{x_{i}\right\}_{1=1}^{\infty}$ and $\left\{y_{l}\right\}_{l=1}^{\infty}$ in $\mathscr{H}$ of norm $\sqrt{k}$ such that

(1) $x_{t} \otimes y_{t} \rightarrow C$ in norm in $\mathscr{C}$,

(2) $x_{i} \otimes s \rightarrow 0$ in norm for any fixed $s \in \mathscr{H}$,

(3) $s \otimes y_{1} \rightarrow 0$ in norm for any fixed $s \in \mathscr{H}$.

If $S$ is an operator as stated above, then the ultraweakly closed algebra $\mathscr{A}$ generated by the polynomials in $S$ has both of these properties 
(see [5] for details). Under the isomorphism, $\Gamma$, that arises naturally in the development of [5], $\Gamma(z)=S$. So $T=S$ in this particular situation, and any results given below for $T$ apply directly to $S$ as well. In fact, all of the results of this paper hold any time $\mathscr{A}$ has property $A_{\kappa_{0}}$ (see [10], Proposition 6.1 and Definitions 2.7 and 2.8).

2. Factorization. We will state and prove the specific form of the factorization that will be needed. Only one row of a matrix factorization will be developed. The proof given here is essentially that of [1] reorganized, and it can be expanded to given a full matricial result for the algebra $\mathscr{A}$ (although the results of [1] apply to more general settings than that used here). The approach given in this note is compartmentalized in order to allow a smoother introduction of a needed modification.

Let $l^{\infty}(\mathscr{H})$ denote the Banach space of all bounded sequences $\left\{x_{i}\right\}_{i=0}^{\infty} \subset \mathscr{H}$ with norm given by $\left\|\left\{x_{i}\right\}_{i=0}^{\infty}\right\| \equiv \sup \left\{\left\|x_{i}\right\|\right\}_{i=0}^{\infty}$. Let $l^{1}(\mathscr{C})$ denote the Banach space of all sequences $\left\{C_{i}\right\}_{i=0}^{\infty} \subset \mathscr{C}$ with norm given by $\left\|\left\{C_{i}\right\}\right\|_{i l=1}^{\infty} \equiv \sum_{i=1}^{\infty}\left\|C_{i}\right\|<\infty$. If $\alpha \in l^{\infty}(\mathscr{H})$, then $\alpha(i)$ will represent the $i$ th term of $\alpha$, i.e., $\alpha=(\alpha(0), \alpha(1), \alpha(2), \ldots)$. Likewise if $\gamma \in l^{1}(C)$, then $\gamma=(\gamma(1), \gamma(2), \gamma(3), \ldots)$. For $n=0,1,2, \ldots$, define $T_{n}: l^{\infty}(\mathscr{H}) \rightarrow$ $l^{\infty}(\mathscr{H})$ by

$$
T_{n}\left(\{\alpha(i)\}_{i=0}^{\infty}\right)=(\alpha(0), \alpha(1), \ldots, \alpha(n), 0,0, \ldots, 0, \ldots)
$$

for all $\alpha \in l^{\infty}(\mathscr{H})$. For $n=1,2, \ldots$, the same symbol, $T_{n}$, will represent an operator $T_{n}: l^{1}(\mathscr{C}) \rightarrow l^{1}(\mathscr{C})$ given by

$$
T_{n}\left(\{\gamma(i)\}_{i=1}^{\infty}\right)=(\gamma(1), \gamma(2), \ldots, \gamma(n), 0,0, \ldots) .
$$

For $c_{0}, c_{1}, \ldots, c_{n} \in \mathbf{R}^{+}$, let $B_{\mathscr{H}}\left(c_{0}, c_{1}, \ldots, c_{n}\right)$ denote $\left\{\alpha \in T_{n}\left(l^{\infty}(\mathscr{H})\right)\right.$ : $\|\alpha(i)\|<c_{i}$ for $\left.i=0,1, \ldots, n\right\}$. Note that if $\alpha \in B_{\mathscr{H}}\left(c_{0}, c_{1}, \ldots, c_{n}\right)$ then $\alpha(j)=0$ for all $j>n$. For $k_{1}, k_{2}, \ldots, k_{n} \in \mathbf{R}^{+}$let $B_{\mathscr{C}}\left(k_{1}, k_{2}, \ldots, k_{n}\right)$ denote $\left\{\gamma \in T_{n}\left(l^{1}(\mathscr{C})\right):\|\gamma(i)\|<k_{i}\right.$ for $\left.i=1,2, \ldots, n\right\}$. Let $l_{F}^{\infty}(\mathscr{H}) \equiv$ $\bigcup_{n=1}^{\infty}\left(T_{n}\left(l^{\infty}(\mathscr{H})\right)\right.$. Finally, define $\Lambda: l_{F}^{\infty}(\mathscr{H}) \rightarrow l^{1}(\mathscr{C})$ by

$$
\Lambda\left(x, y_{1}, y_{2}, \ldots, y_{n}, \ldots\right)=\left(x \otimes y_{1}, x \otimes y_{2}, \ldots, x \otimes y_{n}, \ldots\right)
$$

for $\left(x, y_{1}, y_{2}, \ldots\right) \in l_{F}^{\infty}(\mathscr{H})$. The symbol $T_{n}$ will only be used in Lemma 1 below, so there should be no confusion of $T_{n}$ with the operator $T$. For $M \subset \mathscr{H}$ and $\varepsilon>0$, let $(M: \varepsilon) \equiv\left\{\beta \in l^{\infty}(\mathscr{H}):\langle\beta(0), m\rangle=0\right.$ and $\|\beta(0) \otimes m\|<\varepsilon$ for all $m \in M\}$.

LEMMA 1. Let $n=1,2, \ldots$ If $\alpha \in T_{n}\left(l^{\infty}(\mathscr{H})\right)$, and $k_{1}, k_{2}, \ldots, k_{n} \in$ $\mathbf{R}^{+}$, and $M$ is any fixed finite subset of $\mathscr{H}$, and $\varepsilon>0$, then the closure in $l^{1}(\mathscr{C})$ of

$$
\Lambda\left(\alpha+\left(B_{\mathscr{H}}\left(\sqrt{k_{1}+k_{2}+\cdots+k_{n}}, \sqrt{k_{1}}, \sqrt{k_{2}}, \ldots, \sqrt{k_{n}}\right) \cap(M: \varepsilon)\right)\right)
$$

contains $\Lambda(\alpha)+B_{\mathscr{C}}\left(k_{1}, k_{2}, \ldots, k_{n}\right)$. 
Proof. Assume that the lemma is true as stated for some $n=1,2, \ldots$ We will show that the statement is true when $n$ is replaced with $n+1$. So let $\alpha \in T_{n+1}\left(l^{\infty}(\mathscr{H})\right)$ and let $\gamma \in \Lambda(\alpha)+B_{\mathscr{C}}\left(k_{1}, k_{2}, \ldots, k_{n+1}\right)$. Choose any $\varepsilon>0$, and any finite set $M \subset \mathscr{H}$. We have $T_{n}(\gamma) \in \Lambda\left(T_{n} \alpha\right)+$ $B_{\mathscr{C}}\left(k_{1}, k_{2}, \ldots, k_{n}\right)$. So by the induction hypothesis there exists

$$
\begin{aligned}
\beta \in & B_{\mathscr{H}}\left(\sqrt{k_{1}+k_{2}+\cdots+k_{n}}, \sqrt{k_{1}}, \sqrt{k_{2}}, \ldots, \sqrt{k_{n}}\right) \\
& \cap((M \cup\{\alpha(n+1)\}): \varepsilon / 5)
\end{aligned}
$$

with

$$
\left\|\Lambda\left(\left(T_{n} \alpha\right)+\beta\right)-T_{n} \gamma\right\|<\varepsilon / 5 .
$$

Using the second stated property for the algebra $\mathscr{A}$, choose $x, y \in \mathscr{H}$ with

$$
\begin{gathered}
\|x \otimes y-(\gamma(n+1)-(\alpha(0) \otimes \alpha(n+1)))\|<\varepsilon / 5, \text { and } \\
\|x\|=\|y\|<\sqrt{k_{n+1}}, \text { and }\langle x, \beta(0)\rangle=0, \text { and } \\
\|x \otimes(\alpha(j)+\beta(j))\|<\varepsilon / 5(n+1) \quad \text { for } j=1,2, \ldots, n+1, \quad \text { and } \\
\|(\alpha(0)+\beta(0)) \otimes y\|<\varepsilon / 5 \text { and }(x, 0,0, \ldots, 0, \ldots) \in(M: 4 \varepsilon / 5) .
\end{gathered}
$$

Set

$$
\beta^{\prime}=(\beta(0)+x, \beta(1), \beta(2), \ldots, \beta(n), y, 0, \ldots, 0, \ldots)
$$

Now

$$
\begin{aligned}
& \left\|\Lambda\left(\alpha+\beta^{\prime}\right)-\gamma\right\| \\
& \leq\left\|\Lambda\left(T_{n}\left(\alpha+\beta^{\prime}\right)\right)-T_{n}(\gamma)\right\| \\
& +\|((\alpha(0)+\beta(0)+x) \otimes(\alpha(n+1)+y))-\gamma(n+1)\| \\
& \leq \|(x \otimes(\alpha(1)+\beta(1)), x \otimes(\alpha(2)+\beta(2)), \ldots, x \otimes(\alpha(n)+\beta(n)) \text {, } \\
& 0, \ldots, 0, \ldots) \| \\
& +\left\|\Lambda\left(\left(T_{n} \alpha\right)+\beta\right)-T_{n} \gamma\right\|+\|x \otimes y+(\alpha(0) \otimes \alpha(n+1))-\gamma(n+1)\| \\
& +\|(\alpha(0)+\beta(0)) \otimes y\|+\|\beta(0) \otimes \alpha(n+1)\|+\|x \otimes \alpha(n+1)\| \\
& <\frac{\varepsilon}{5(n+1)} n+\frac{\varepsilon}{5}+\frac{\varepsilon}{5}+\frac{\varepsilon}{5}+\frac{\varepsilon}{5}+\frac{\varepsilon}{5(n+1)}=\varepsilon \text {. }
\end{aligned}
$$

We are done since

$$
\beta^{\prime} \in B_{\mathscr{H}}\left(\sqrt{k_{1}+k_{2}+\cdots+k_{n+1}}, \sqrt{k_{1}}, \sqrt{k_{2}}, \ldots, \sqrt{k_{n+1}}\right) \cap(M: \varepsilon) .
$$


If $C \in \mathscr{C}$ and $A \in \mathscr{A}$, then it is possible to define $A C \in \mathscr{C}$ by $[B](A C) \equiv[B A](C)$ for all $B \in \mathscr{A}$. Now let $\mathscr{N}$ denote $\left\{C \in \mathscr{C}: A^{n} C=0\right.$ for some $n=0,1,2, \ldots\}$. Recall $T=\Gamma(z) \in \mathscr{A}$. It is easy to verify using $\Gamma$ that $\mathscr{N}$ is norm dense in $\mathscr{C}$.

In the following theorem, the result obtained by just observing statements (i) and (ii) gives the standard factorization result fitting this situation (again see [1] for the complete matricial form of the result). This by itself is sufficient to yield analytic subspaces, except that there is no guarantee that the map associating each $y \in \mathscr{S}$ (see Theorem 2) with $f_{v} \in H(U)$ is one to one. Statements (iii) and (iv) below involve a result that will later be used to establish the needed injectivity.

THEOREM 1. Given $\gamma \in l^{1}(\mathscr{C})$ and $\varepsilon_{i}>0, i=1,2, \ldots$, there exists $\alpha \in l^{\infty}(\mathscr{H})$ with

(i) $(\alpha(0) \otimes \alpha(2 i-1))=\gamma(i)$ for $i=1,, \ldots$,

(ii) $\|\alpha(2 i-1)\|<\sqrt{\|\gamma(i)\|}+\varepsilon_{i}$ for $i=1,2, \ldots$,

(iii) $(\alpha(0) \otimes \alpha(2 i)) \in \mathscr{N}$ for $i=1,2, \ldots$,

(iv) $\{\alpha(2 i): i=1,2, \ldots\}$ norm dense in the unit ball of $\mathscr{H}$.

Proof. Let $\left\{r_{i}\right\}_{i=1}^{\infty}$ be any countable dense subset of the unit ball of $\mathscr{H}$. The proof will consist of finding a certain sequence $\left\{\alpha_{n}\right\}_{n=1}^{\infty} \subset l^{\infty}(\mathscr{H})$ for which $\left\{\alpha_{n}(i)\right\}_{n=1}^{\infty}$ is Cauchy for each $i=0,1,2, \ldots$. This sequence will be defined by induction, and its "adherent" point $\alpha$ will turn out to satisfy the statement of the theorem. We assume without loss of generality that $\sum_{i=1}^{\infty} \varepsilon_{i}<\infty$. Let $k_{i}=\|\gamma(i)\|, i=1,2, \ldots$.

To begin the induction define $\hat{\beta}_{1} \in l^{1}(\mathscr{C})$ by $\hat{\beta}_{1}=(\gamma(1), 0$, $0, \ldots, 0, \ldots)$.

By Lemma 1 , choose $\hat{\alpha}_{1} \in B_{\mathscr{H}}\left(\sqrt{k_{1}}, \sqrt{k_{1}}\right)$ with

$$
\left(\Lambda\left(\hat{\alpha}_{1}\right)-\hat{\beta}_{1}\right) \in B_{\mathscr{C}}\left(\varepsilon_{1}^{2} / 4\right) .
$$

Next choose $R_{1} \in \mathscr{N}$ with

$$
\left\|\hat{\alpha}_{1}(0) \otimes r_{1}-R_{1}\right\|<\varepsilon_{1}^{2} / 4 \text {. }
$$

Set $\alpha_{1}=\left(\hat{\alpha}_{1}(0), \hat{\alpha}_{1}(1), r_{1}, 0,0, \ldots\right)$ and set $\beta_{1}=\left(\gamma(1), R_{1}, 0,0, \ldots\right)$. We have

$$
\left(\Lambda\left(\alpha_{1}\right)-\beta_{1}\right) \in B_{\mathscr{C}}\left(\varepsilon_{1}^{2} / 4, \varepsilon_{1}^{2} / 4\right) .
$$

Now suppose that $\alpha_{1}, \alpha_{2}, \ldots, \alpha_{n} \in l^{\infty}(\mathscr{H})$ and $R_{1}, R_{2}, \ldots, R_{n} \in \mathscr{N}$ have been chosen so that

$$
* \quad\left(\Lambda\left(\alpha_{n}\right)-\beta_{n}\right) \in B_{\mathscr{C}}\left(\frac{\varepsilon_{1}^{2}}{4^{n}}, \frac{\varepsilon_{1}^{2}}{4^{n}}, \frac{\varepsilon_{2}^{2}}{4^{n-1}}, \frac{\varepsilon_{2}^{2}}{4^{n-1}}, \ldots, \frac{\varepsilon_{n}^{2}}{4}, \frac{\varepsilon_{n}^{2}}{4}\right)
$$


where

$$
\beta_{n} \equiv\left(\gamma(1), R_{1}, \gamma(2), R_{2}, \ldots, \gamma(n), R_{n}, 0,0, \ldots\right) \in l^{1}(\mathscr{C}) .
$$

The procedure for choosing $\alpha_{n+1}$ and $R_{n+1}$ will be given. Set

$$
\hat{\beta}_{n+1}=\left(\gamma(1), R_{1}, \gamma(2), R_{2}, \ldots, \gamma(n), R_{n}, \gamma(n+1), 0,0, \ldots\right) \text {. }
$$

By Lemma 1 choose

$$
\left.\begin{array}{r}
\hat{\alpha}_{n+1} \in \alpha_{n}+\beta_{\mathscr{H}}\left(\sqrt{\frac{\varepsilon_{1}^{2}}{4^{n}}+\frac{\varepsilon_{1}^{2}}{4^{n}}+\cdots+\frac{\varepsilon_{n}^{2}}{4}+\frac{\varepsilon_{n}^{2}}{4}+k_{n+1}},\right. \\
\frac{\varepsilon_{1}}{2^{n}}, \frac{\varepsilon_{1}}{2^{n}}, \ldots, \frac{\varepsilon_{n}}{2}, \frac{\varepsilon_{n}}{2}, \sqrt{k_{n+1}}
\end{array}\right)
$$

such that

$$
\left(\Lambda\left(\hat{\alpha}_{n+1}\right)-\hat{\beta}_{n+1}\right) \in B_{\mathscr{C}}\left(\frac{\varepsilon_{1}^{2}}{4^{n+1}}, \frac{\varepsilon_{1}^{2}}{4^{n+1}}, \ldots, \frac{\varepsilon_{n}^{2}}{4^{2}}, \frac{\varepsilon_{n}^{2}}{4^{2}}, \frac{\varepsilon_{n+1}^{2}}{4}\right)
$$

and

$$
\left(\hat{\alpha}_{n+1}(0)-\alpha_{n}(0)\right) \perp \alpha_{i}(0) \text { for } i=1,2, \ldots, n .
$$

Now choose $R_{n+1} \in \mathscr{N}$ with

$$
\left\|R_{n+1}-\hat{\alpha}_{n+1}(0) \otimes r_{n+1}\right\|<\varepsilon_{n+1}^{2} / 4 .
$$

Set

$$
\beta_{n+1}=\left(\gamma(1), R_{1}, \gamma(2), R_{2}, \ldots, \gamma(n+1), R_{n+1}, 0,0, \ldots\right)
$$

and

$$
\alpha_{n+1}=\left(\hat{\alpha}_{n+1}(0), \hat{\alpha}_{n+1}(1), \ldots, \hat{\alpha}_{n+1}(2 n+1), r_{n+1}, 0,0, \ldots\right)
$$

So

$$
\left(\Lambda\left(\alpha_{n+1}\right)-\beta_{n+1}\right) \in B_{\mathscr{C}}\left(\frac{\varepsilon_{1}^{2}}{4^{n+1}}, \frac{\varepsilon_{1}^{2}}{4^{n+1}}, \ldots, \frac{\varepsilon_{n+1}^{2}}{4}, \frac{\varepsilon_{n+1}^{2}}{4}\right)
$$

and

$$
\left(\alpha_{n+1}(0)-\alpha_{n}(0)\right) \perp \alpha_{i}(0) \text { for } i=1,2, \ldots, n \text {. }
$$

Also

$$
\begin{array}{r}
\left(\alpha_{n+1}-\alpha_{n}\right) \in B_{\mathscr{H}}\left(\sqrt{\frac{\varepsilon_{1}^{2}}{4^{n}}+\frac{\varepsilon_{1}^{2}}{4^{n}}+\cdots+\frac{\varepsilon_{n}^{2}}{4}+\frac{\varepsilon_{n}^{2}}{4}+k_{n+1}},\right. \\
\left.\frac{\varepsilon_{1}}{2^{n}}, \frac{\varepsilon_{1}}{2^{n}}, \ldots, \frac{\varepsilon_{n}}{2}, \frac{\varepsilon_{n}}{2}, \sqrt{k_{n+1}}, 2\left\|r_{n+1}\right\|\right) .
\end{array}
$$


For each $i=0,1,2, \ldots$, set $\alpha(i)=\lim _{n \rightarrow \infty} \alpha_{n}(i)$. It is clear that this limit exists when $i=0$, since $\left(\alpha_{n+1}(0)-\alpha_{n}(0)\right) \perp\left(\alpha_{j}(0)-\alpha_{j-1}(0)\right)$ for $j=2,3, \ldots, n$. It is also trivial to show that the limit exists for $i=1,2, \ldots$, and that $\|\alpha(2 i-1)\|<\sqrt{k_{i}}+\varepsilon_{i}$. By definition of each $\beta_{n}, n=1,2, \ldots$, it follows that (i) and (iii) hold. Condition (iv) has also been met. We are done.

REMARK. It is actually possible to choose $\alpha(0)$ arbitrarily close to any preassigned vector $x \in \mathscr{H}$ provided that one is willing to give up the inequality of (ii) in the statement for the indices $i=1,2, \ldots, n$ where $n$ depends on $\|x-\alpha(0)\|$. (The inequality of (ii) will still hold for all other indices). To show this, first note that $x \otimes x \in \mathscr{C}$ is arbitrarily close to a vector $C \in \mathscr{C}$ which is cyclic under the action of $T$ on $\mathscr{C}$. Then there exist $x^{\prime}, y \in \mathscr{H}$ with $\left\|x-x^{\prime}\right\|$ arbitrarily small and $x^{\prime} \otimes y=C$. For any given $n=1,2, \ldots$ choose polynomials $p_{1}, p_{2}, \ldots, p_{n}$ such that

$$
\left\|p_{i}(T) x^{\prime} \otimes y-\gamma(i)\right\|<4^{1-n-i} \varepsilon^{2}
$$

for $i=1,2, \ldots, n$. Then set $y_{l}=\left(p_{i}(T)\right)^{*} y$ for $i=1,2, \ldots, n$, and set

$$
\alpha_{n}=\left(x^{\prime}, y_{1}, r_{1}, y_{2}, r_{2}, \ldots, y_{n}, r_{n}, 0,0, \ldots\right) \text {. }
$$

Then $\alpha_{n}$ satisfies $*$ provided $R_{1}, R_{2}, \ldots, R_{n}$ are chosen appropriately. Now run the induction process from here (i.e., $*$ ) on out. If $n$ is chosen sufficiently large, then $\|x-\alpha(0)\|$ can be made as small as desired.

3. Full analytic invariant subspaces. Next the main theorem will be given. Its proof has been simplified using a suggestion made by $\mathrm{H}$. Bercovici.

THEOREM 2. The operator $T \in \mathscr{A}$ has a nontrivial closed invariant subspace that is full analytic.

Proof. For $n=0,1,2, \ldots$, define $C_{n}$ to be that unique element in $\mathscr{C}$ for which $\left[T^{m}\right]\left(C_{n}\right)=\delta_{m, n}$ where $\delta_{m, n}$ represents the Kronecker delta. Set

$$
C=\left(C_{0}, C_{1}, \frac{1}{2^{2}} C_{2}, \ldots, \frac{1}{i^{2}} C_{i}, \ldots\right) \in l^{1}(\mathscr{C}) .
$$

For $i=1,2, \ldots$, set $\varepsilon_{i+1}=1 / i$. Set $\varepsilon_{1}=1$. Apply Theorem 1 to obtain $\left(x, y_{1}, y_{1}^{\prime}, y_{2}, y_{2}^{\prime}, \ldots\right) \in l^{\infty}(\mathscr{H})$ with $x \otimes y_{i+1}=\left(1 / i^{2}\right) C_{i}$ for $i=1,2, \ldots$, and $\left\|y_{i+1}\right\| \leq \sqrt{\left(1 / i^{2}\right)}+1 / i=2 / i$, and $x \otimes y_{1}=C_{0}$, and $x \otimes y_{i}^{\prime} \in \mathscr{N}$ for $i=1,2, \ldots$, and $\left\{y_{l}^{\prime}\right\}_{i=1}^{\infty}$ dense in $B_{\mathscr{H}}$. 
Now let $\mathscr{S}=\overline{\mathscr{A} x}$, and let $P: \mathscr{H} \rightarrow \mathscr{S}$ be the orthogonal projection. For $i=1,2, \ldots$ define $\hat{y}_{i+1} \equiv P\left(i^{2} y_{i+1}\right)$, and define $\hat{y}_{1}=P y_{1}$. For $y \in \mathscr{S}$ define a function $f_{y}$ on $U$ by

$$
f_{y}(z)=\sum_{i=0}^{\infty}\left\langle y, \hat{y}_{i+1}\right\rangle z^{l} \quad \text { for all } z \in U
$$

This series converges for all $z \in U$ since

$$
\begin{aligned}
\lim \sup \left|\left\langle y, \hat{y}_{i+1}\right\rangle\right|^{1 / i} & \leq \lim \sup \left\|\hat{y}_{i+1}\right\|^{1 / i} \leq \lim \sup \left\|i^{2} y_{i+1}\right\|^{1 / i} \\
& \leq \lim \sup |2 i|^{1 / i}=1 .
\end{aligned}
$$

Next we show that $P T^{*} \hat{y}_{i}=\hat{y}_{i-1}$ for any fixed $i=2,3, \ldots$ Now for $m=1,2, \ldots$,

$$
\left[T^{m}\right]\left(x \otimes P T^{*} \hat{y}_{i}\right)=\left\langle T^{m+1} x, \hat{y}_{i}\right\rangle=\delta_{i-1, m+1}=\left[T^{m}\right]\left(x \otimes \hat{y}_{i-1}\right) .
$$

So $x \otimes P T^{*} \hat{y}_{i}=x \otimes \hat{y}_{i-1}$. Or $x \otimes\left(P T^{*} \hat{y}_{i}-\hat{y}_{i-1}\right)=0$. Since $P T^{*} \hat{y}_{i}-$ $\hat{y}_{i-1} \in \mathscr{S}=\overline{\mathscr{A} x}$, it follows that $P T^{*} \hat{y}_{l}=\hat{y}_{i-1}$.

Using the fact that has just been verified in the preceding paragraph, it is easy to show that $f_{T y}(z)=z f_{y}(z)$ for all $y \in \mathscr{S}$ and $z \in U$.

Next it has to be shown that the map $y \rightarrow f_{y}$ is one to one. First of all, for each $\lambda \in U$ there is $y_{\lambda} \in \mathscr{S}$ such that $\left\langle y, y_{\lambda}\right\rangle=f_{y}(\lambda)$ for all $y \in \mathscr{S}$. In fact for $\lambda \in U$,

$$
y_{\lambda}=\sum_{i=0}^{\infty} \lambda^{i} \hat{y}_{i+1}
$$

It is easily seen that if $\lambda_{i} \rightarrow \lambda \in U$, then $y_{\lambda_{l}} \rightarrow y_{\lambda}$ in norm. This implies that if $\left\{s_{l}\right\}_{i=1}^{\infty} \subset \mathscr{S}$ is a bounded sequence with $f_{s_{i}} \rightarrow 0$ pointwise on $U$, then $f_{s_{t}} \rightarrow 0$ uniformly on compact subsets of $U$.

Now suppose $y \in \mathscr{S}$, with $f_{y}$ identically zero on $U$. Then there exists a sequence of polynomials $\left\{p_{j}\right\}_{j=1}^{\infty}$ with $p_{j}(T) x$ converging to $y$ in norm. However, $f_{p_{j}(T) x}=p_{j} f_{x}$ and $f_{p_{j}(T) x} \rightarrow f_{y}$ pointwise, so $p_{j} \rightarrow 0$ uniformly on compact subsets of $U$. Now suppose that $\|y\| \neq 0$. A contradiction will be found. It can be assumed without loss of generality that $\|y\|=1$. Choose $i_{0}=1,2, \ldots$ such that $\left\|y-y_{i_{0}}^{\prime}\right\|<1 / 10$ (recall the $y_{i}^{\prime}$ were found using Theorem 1 earlier). There exists a positive integer $m$ such that $T^{m} x \otimes y_{i_{0}}^{\prime}=0$. Choose $j_{0}$ so large that

$$
p_{J_{0}}=z^{m} p+q \quad \text { where } p \text { and } q \text { are polynomials with }
$$

$$
\sup \{|q(\lambda)|: \lambda \in U\}<\frac{1}{11} \frac{1}{\|x\|}
$$


and

$$
\left\|p_{J_{0}}(T) x-y\right\|<1 / 10 \quad\left(\text { so }\left\|p_{j_{0}}(T) x\right\|<2\right) .
$$

Then by (1)

$$
\begin{aligned}
\left\|\left(p_{j_{0}}(T) x\right) \otimes y_{i_{0}}^{\prime}\right\| & =\left\|\left(T^{m} p(T) x\right) \otimes y_{i_{0}}^{\prime}+(q(T) x) \otimes y_{i_{0}}^{\prime}\right\| \\
& =\left\|(q(T) x) \otimes y_{i_{0}}^{\prime}\right\|<\frac{1}{11\|x\|}\|x\| \frac{11}{10}<\frac{1}{10}
\end{aligned}
$$

Thus by (2), $\left\|\left(p_{J_{0}}(T) x\right) \otimes y\right\|<3 / 10$ since $\left\|y-y_{i_{0}}^{\prime}\right\|<1 / 10$, and thus $\|y \otimes y\|<4 / 10$ also by (2). This is impossible since $\|y \otimes y\|=\|y\|^{2}=1$. The proof is complete.

REMARK. The theorem can be easily amended using the remark made after Theorem 1 to show that the element $x$ which generates the full analytic subspace can be chosen arbitrarily close to any given element in the Hilbert space.

It should be noted that in certain cases it is highly desirable to find maps $y \rightarrow f_{v}$ which are not one to one for which $f_{T y}(z)=z f_{y}$. For if $T$ is invertible, then $\left\{y: f_{y} \equiv 0\right.$ on $\left.U\right\}$ is closed and invariant for $T$ and $T^{-1}$. The existence of such subspaces is directly connected to the invariant subspace problem for operators that have the same norm and spectral radius [6].

Finally the reflexivity results of [2] will be shown to follow from Theorem 2 above for the operator $T$. The proof uses ideas similar to those established for subnormal operators in [8].

THEOREM 3.The algebra $\mathscr{A}$ is reflexive.

Proof. It has to be shown that if $A \mathscr{M} \subset \mathscr{M}$ for all $\mathscr{M} \subset \mathscr{H}$ invariant under $\mathscr{A}$, then $A \in \mathscr{A}$. The proof of Theorem 2 can be amended to show that $\mathscr{R} \equiv\{x \in \mathscr{H}: \overline{\mathscr{A} x}$ is full analytic $\}$ is norm dense in $\mathscr{H}$. If $x \in \mathscr{R}$, then $\overline{\mathscr{A} x}$ is invariant under $A$. It is not hard to show that there exists $f \in H^{\infty}$ with $f(T) y=A y$ for all $y \in \overline{\mathscr{A} x}$. If it can be shown that given any $x_{1} \in \mathscr{R}$ we have $f(T) y=A y$ for all $y \in \overline{\mathscr{A} x_{1}}$, then by the density of $\mathscr{R}$ it will follow that $A=f(T)$ on all of $\mathscr{H}$.

So consider $\overline{\mathscr{A}\left(x_{1}+x\right)}$, and set $B=A-f(T)$. Then $B\left(x+x_{1}\right)=$ $B x_{1}$. Now there exists some $g \in H^{\infty}$ with $g(T) y=B y$ for all $y \in \overline{\mathscr{A} x_{1}}$. So $B x_{1}=B\left(x+x_{1}\right)=g(T) x_{1} \in \overline{\mathscr{A}\left(x+x_{1}\right)}$. Therefore there exists a 
sequence $\left\{p_{i}\right\}_{i=1}^{\infty}$ of polynomials with

$$
p_{i}(T)\left(x+x_{1}\right) \rightarrow g(T) x_{1}
$$

in norm. This equation shows that $p_{i}(T) B x_{1} \rightarrow g(T) B x_{1}$ in norm, so $p_{1} \rightarrow g$ pointwise on $U$. This equation also gives $p_{i}(T) g(T)\left(x+x_{1}\right) \rightarrow$ $g(T) B x_{1}$ in norm. So $p_{i}(T) g(T) x \rightarrow 0$ in norm. But $\mathscr{A} x$ is full analytic so $p, g \rightarrow 0$ pointwise in $U$. This means $g=0$, and therefore that $B=0$ on $\overline{\mathscr{A} x_{1}}$. The result follows.

\section{REFERENCES}

[1] H. Bercovici, C. Foias, and C. Pearcy, A matricial factorization theorem and the structure of (BCP)-operators, (to appear).

[2] H. Bercovici, C. Foias, J. Langsam, and C. Pearcy, ( $B C P)$-operators are reflexive, Michigan Math. J., 29 (1982), 371-379.

[3] S. Brown, Banach algebras that are dual spaces, Ph. D. Thesis, University of California at Santa Barbara, 1978.

[4] Some invariant subspaces for subnormal operators, Integral Equations and Operator Theory, 1 (1978), 310-333.

[5] S. Brown, B. Chevreau, and C. Pearcy, Contractions with rich spectrum have invariant subspaces, J. Operator Theory, 1 (1979), 123-126.

[6] C. Foias and C. Pearcy, (BCP)-operators and enrichment of invariant subspace lattices, J. Operator Theory, 9 (1983), 187-202.

[7] R. Olin and J. Thomson, Algebras of subnormal operators, J. Functional Anal., 37 (1980), 271-301.

[8] Cellular-indecomposable subnormal operators, to appear.

[9] J. Sheung, On the preduals of certain operator algebras, Ph. D. Dissertation, University of Hawaii, 1983.

[10] H. Bercovici, C. Foias, C. Pearcy, Dual algebras with applications to invariant subspaces and dilation theory, Conference Board of Mathematics 56, 1985.

Received May 6, 1985 and in revised form August 20, 1986.

UNIVERSITY OF HAWAII

HONOLULU, HI 96822

Present address: University of Michigan

Ann Arbor, MI 48109 


\section{PACIFIC JOURNAL OF MATHEMATICS \\ EDITORS}

V. S. VARADARAJAN

(Managing Editor)

University of California

Los Angeles, CA 90024

Herbert Clemens

University of Utah

Salt Lake City, UT 84112

R. FINN

Stanford University

Stanford, CA 94305
HERMANN FLASCHKA

University of Arizona

Tucson, AZ 85721

RAMESH A. GANGOLLI

University of Washington Seattle, WA 98195

VAUGHAN F. R. JONES

University of California

Berkeley, CA 94720
ROBION KIRBY

University of California

Berkeley, CA 94720

C. C. MOORE

University of California

Berkeley, CA 94720

HAROLD STARK

University of California, San Diego

La Jolla, CA 92093

\section{ASSOCIATE EDITORS}
R. ARENS
E. F. BECKENBACH
B. H. NEUMANN
F. WOLF
K. YOSHIDA
(1906-1982)

\section{SUPPORTING INSTITUTIONS}

UNIVERSITY OF ARIZONA

UNIVERSITY OF BRITISH COLUMBIA

CALIFORNIA INSTITUTE OF TECHNOLOGY

UNIVERSITY OF CALIFORNIA

MONTANA STATE UNIVERSITY

UNIVERSITY OF NEVADA, RENO

NEW MEXICO STATE UNIVERSITY

OREGON STATE UNIVERSITY
UNIVERSITY OF OREGON UNIVERSITY OF SOUTHERN CALIFORNIA

STANFORD UNIVERSITY

UNIVERSITY OF HAWAII

UNIVERSITY OF TOKYO

UNIVERSITY OF UTAH

WASHINGTON STATE UNIVERSITY

UNIVERSITY OF WASHINGTON 


\section{Pacific Journal of Mathematics \\ Vol. 132, No. $1 \quad$ January, 1988}

Scott W. Brown, Full analytic subspaces for contractions with rich spectrum ...1

Robert Main Burton, Jr. and Tae-Sung Kim, An invariance principle for

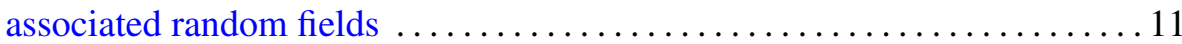

Ana M. Viola-Prioli and Jorge Viola-Prioli, Rings whose kernel functors

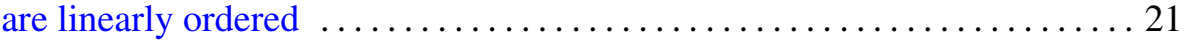

David E. Handelman, Representing polynomials by positive linear

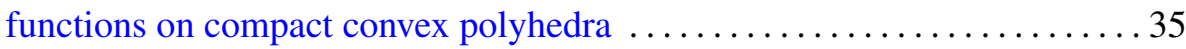

Patrick Keef, On the Tor functor and some classes of abelian groups . .....66

Dennis R. Malm, Simplicity of partial and Schmidt differential operator

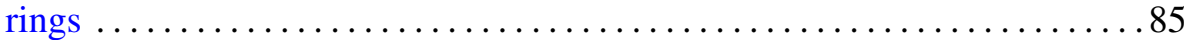

José M. Montesinos and Carmen Safont, On the Birman invariants of

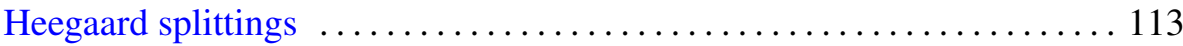

N. P. Mukherjee and Prabir Bhattacharya, The normal index of a finite group

Mario Petrich and Norman R. Reilly, Semigroups generated by certain operators on varieties of completely regular semigroups

Robert Tijdeman and Lian Xiang Wang, Sums of products of powers of given prime numbers

Joel Larry Weiner, First integrals for a direction field on a simply connected plane domain 